Journal of Applied Analysis

Vol. 6, No. 1 (2000), pp. 149-157

\title{
ON LINEAR DEPENDENCE OF ITERATES
}

\author{
J. MATKOWSKI and W. ZHANG
}

Received September 9, 1997 and, in revised form, February 1, 2000

\begin{abstract}
A functional equation related to a problem of linear dependence of iterates is considered.
\end{abstract}

\section{Introduction}

The polynomial-like iterative functional equation

$$
\lambda_{0} f^{0}(x)+\lambda_{1} f^{1}(x)+\ldots+\lambda_{n} f^{n}(x)=F(x), \quad x \in \mathbf{X},
$$

where $\mathbf{X}$ stands for a real or complex linear space and $f^{k}$ denotes the $k$-th iterate of the unknown function $f: \mathbf{X} \rightarrow \mathbf{X}$, i.e., $f^{0}(x)=x$ for $x \in \mathbf{X}$ and $f^{k+1}=f \circ f^{k}$ (here "०" denotes the composition of functions) is discussed extensively, cf. [1]-[11]. An important special case of this equation is

$$
f^{n}(x)=a_{n-1} f^{n-1}(x)+a_{n-2} f^{n-2}(x)+\ldots+a_{0} x, \quad x \in \mathbf{X},
$$

where $a_{0}, \ldots a_{n-1}$ are real or complex numbers. This functional equation can be interpreted as linear dependence of iterates of $f$. In 1974 Nabeya [8] discussed (1) for $n=2$ and $\mathbf{X}=\mathbf{R}$ in detail by considering its characteristic equation. However Nabeya's idea appears to be difficult to apply in solving equation (1) for $n \geq 3$. During the 26th International Symposium on Functional Equations held in Spain in 1988 the first author presented the result [6] that the solutions of (1) for $n=k$ are solutions of (1) for

1991 Mathematics Subject Classification. 39B12.

Key words and phrases. Iterate, functional equation, characteristic polynomial.

ISSN 1425-6908 (c) Heldermann Verlag. 
$n=m, \quad m \geq k$, if the characteristic polynomial of the lower order equation exactly divides that of the higher order one. This statement establishes a useful relation in the class of iterative equations of type (1), but until now the proof was not published. In this paper an elementary proof is presented. Furthermore, based on this result some conclusions how the solutions to be ruled by the roots of the relevant characteristic polynomials are given.

\section{Characteristic equations}

Following Euler's idea for differential equations, we formally consider a linear solution

$$
f(x)=r x, \quad x \in \mathbf{X},
$$

of the equation (1) where $r \in \mathbf{C}$ is indeterminate. From (1) we have

$$
r^{n}-a_{n-1} r^{n-1}-\ldots-a_{1} r-a_{0}=0 .
$$

Here (2) is called the characteristic equation of equation (1), its roots are called the characteristic roots, and the left-hand side of (2), denoted by $P_{n}(r)$, is called the characteristic polynomial of equation (1). By the well known relations between roots and coefficients of polynomials equation (1) is equivalent to

$$
f^{n}(x)-\left(\sum_{i=1}^{n} r_{i}\right) f^{n-1}(x)+\left(\sum_{i<j}^{n} r_{i} r_{j}\right) f^{n-2}(x)+\ldots+(-1)^{n} r_{1} r_{2} \ldots r_{n} x=0
$$

for $x \in \mathbf{X}$, where $r_{1}, r_{2}, \ldots, r_{n}$ are $n$ complex roots of the polynomial $P_{n}$. Let $F_{n}\left(r_{1}, r_{2}, \ldots, r_{n}\right) f$ denote the function of the left-hand side of $(3)$ and call it $n$-form of (3). The $n$-form is uniquely determined by given $r_{1}, r_{2}, \ldots, r_{n} \in \mathbf{C}$.

Lemma 1. For fixed $r_{1}, r_{2}, \ldots, r_{n+1} \in \mathbf{C}$, if $F_{n}\left(r_{1}, r_{2}, \ldots, r_{n}\right) f=0$ then

$$
F_{n+1}\left(r_{1}, \ldots, r_{n}, r_{n+1}\right) f=0 .
$$

Proof. Since $F_{n}\left(r_{1}, r_{2}, \ldots, r_{n}\right) f=0$, i.e., $f$ satisfies equation (3), we have

$$
\begin{array}{r}
f^{n+1}(x)=f^{n}(f(x))=\left(\sum_{i=1}^{n} r_{i}\right) f^{n}(x)-\left(\sum_{i<j}^{n} r_{i} r_{j}\right) f^{n-1}(x)+\ldots \\
+(-1)^{n+1} r_{1} r_{2} \ldots r_{n} f(x), \quad x \in \mathbf{X} .
\end{array}
$$


Thus, for all $x \in \mathbf{X}$, the $(n+1)$-form satisfies

$$
\begin{aligned}
& F_{n+1}\left(r_{1}, \ldots, r_{n}, r_{n+1}\right) f(x) \\
& =f^{n+1}(x)-\left(\sum_{i=1}^{n+1} r_{i}\right) f^{n}(x)+\left(\sum_{i<j}^{n+1} r_{i} r_{j}\right) f^{n-1}(x)+\ldots+(-1)^{n+1} r_{1} r_{2} \ldots r_{n+1} x \\
& =\left(\sum_{i=1}^{n} r_{i}-\sum_{i=1}^{n+1} r_{i}\right) f^{n}(x)-\left(\sum_{i<j}^{n} r_{i} r_{j}-\sum_{i<j}^{n+1} r_{i} r_{j}\right) f^{n-1}(x) \\
& +\ldots+(-1)^{n+1} r_{1} r_{2} \ldots r_{n+1} x \\
& =-r_{n+1} f^{n}(x)+r_{n+1}\left(\sum_{i=1}^{n} r_{i}\right) f^{n-1}(x)-r_{n+1}\left(\sum_{i<j}^{n} r_{i} r_{j}\right) f^{n-2}(x) \\
& +\ldots+(-1)^{n+1} r_{1} r_{2} \ldots r_{n+1} x=-r_{n+1} F_{n}\left(r_{1}, r_{2}, \ldots, r_{n}\right) f(x)=0 .
\end{aligned}
$$

Now we can prove the result presented in [6].

Theorem 1. Suppose that

$$
\begin{gathered}
Q(r)=r^{k}-b_{k-1} r^{k-1}-\ldots-b_{1} r-b_{0}, \\
P(r)=r^{n}-a_{n-1} r^{n-1}-\ldots-a_{1} r-a_{0},
\end{gathered}
$$

are polynomials, where $r \in \mathbf{C}, k \leq n$, and that $Q \mid P$, i.e., $P$ is exactly divided by $Q$. If a function $f: \mathbf{X} \rightarrow \mathbf{X}$ satisfies the functional equation

$$
f^{k}(x)=b_{k-1} f^{k-1}(x)+b_{k-2} f^{k-2}(x)+\ldots+b_{0} x, \quad x \in \mathbf{X},
$$

then $f$ satisfies functional equation (1), i.e.,

$$
f^{n}(x)=a_{n-1} f^{n-1}(x)+a_{n-2} f^{n-2}(x)+\ldots+a_{0} x, \quad x \in \mathbf{X} .
$$

Proof. Let $r_{1}, r_{2}, \ldots, r_{n}$ be complex roots of $P$. Since $Q \mid P$ we may assume without any loss of generality that $r_{1}, \ldots, r_{k}, \quad k \leq n$, are roots of $Q$. From the definition of $F_{k}$ and (4) we have

$$
F_{k}\left(r_{1}, r_{2}, \ldots, r_{k}\right) f=0 .
$$

By Lemma 1, the function $f$ also satisfies

$$
F_{k+1}\left(r_{1}, \ldots, r_{k}, r_{k+1}\right) f=0 .
$$

Thus, by induction, we can prove easily that

$$
F_{n}\left(r_{1}, r_{2}, \ldots, r_{n}\right) f=0,
$$

that is, $f$ satisfies equation (1). 
Remark 1. Equation (1) of order $n$ has a solution which does not satisfy the equation (4) of order $k$ if $Q \mid P$ but $Q \neq P$.

In fact, if all roots $r_{1}, r_{2}, \ldots, r_{n}$ of $P$ are real and only $r_{1}, r_{2}, \ldots, r_{k}, \quad k<n$, are roots of $Q$, then $f(x)=r_{i} x, \quad x \in \mathbf{X}, \quad i=k+1, \ldots, n$, satisfies (1) but is not a solution of (4).

Remark 2. Let $\mathbf{X}=\mathbf{R}$ and suppose that the coefficients in equation (1) are real. If $r_{0}$ is a complex root of the characteristic polynomial $P_{n}$ with imaginary part $\Im r_{0} \neq 0$, then all solutions of the real 2-order iterative equation

$$
f^{2}(x)=2 \Re r_{0} f(x)-\left|r_{0}\right|^{2} x,
$$

where $\Re r_{0}$ denotes the real part of $r_{0}$ and $\left|r_{0}\right|$ denotes the modulus of $r_{0}$, satisfy equation (1).

This assertion is a consequence of Theorem 1 and the fact that the conjugacy $\bar{r}_{0}$ of $r_{0}$ is also a root of $P_{n}$.

\section{Iterations of solutions}

For convenience, let $F_{n-1}\left(r_{1}, \ldots, \check{r_{k}}, \ldots, r_{n}\right) f$ represent the $(n-1)$-form of (3) determined by $n-1$ characteristic roots $r_{1}, \ldots, r_{k-1}, r_{k+1}, \ldots, r_{n}$.

Theorem 2. Suppose that the characteristic polynomial $P_{n}$ in (2) has $n$ pairwise different roots $r_{1}, \ldots, r_{n}$ and that $f: \mathbf{X} \rightarrow \mathbf{X}$ is a solution of fuctional equation (1). Then for any integer $m \geq 0$,

$$
f^{n+m}=\frac{A_{11}}{\Delta} r_{1}^{m+1} g_{1}+\frac{A_{21}}{\Delta} r_{2}^{m+1} g_{2}+\ldots+\frac{A_{n 1}}{\Delta} r_{n}^{m+1} g_{n},
$$

where

$$
g_{k}:=F_{n-1}\left(r_{1}, \ldots, \check{r_{k}}, \ldots, r_{n}\right) f, \quad k=1,2, \ldots, n,
$$

and $\Delta$ and $A_{k 1}, \quad k=1,2, . ., n$, denote respectively the determinant and algebraic adjuncts of the matrix

$$
A=\left(\begin{array}{llll}
1-\sum_{i \neq 1} r_{i} & \sum_{i<j, \neq 1} r_{i} r_{j} & \ldots & (-1)^{n-1} r_{2} r_{3} \ldots r_{n} \\
1-\sum_{i \neq 2} r_{i} & \sum_{i<j, \neq 2} r_{i} r_{j} & \ldots & (-1)^{n-1} r_{1} r_{3} \ldots r_{n} \\
\ldots . & \ldots & \ldots & \\
1-\sum_{i \neq n} r_{i} & \sum_{i<j, \neq n} r_{i} r_{j} & \ldots & (-1)^{n-1} r_{1} r_{2} \ldots r_{n-1}
\end{array}\right) .
$$

Here $\sum_{i \neq 1}$ and its like denote the summations with respect to the indexes from 1 to $n$ with some shown restriction. 
Proof. Write equation (3) in the equivalent form

$$
\begin{gathered}
f^{n}-\left(\sum_{i \neq n} r_{i}\right) f^{n-1}+\left(\sum_{i<j, \neq n} r_{i} r_{j}\right) f^{n-2}+\ldots+(-1)^{n-1} r_{1} r_{2} \ldots r_{n-1} f \\
=r_{n} f^{n-1}-r_{n}\left(\sum_{i \neq n} r_{i}\right) f^{n-2}+\ldots+(-1)^{n-1} r_{1} r_{2} \ldots r_{n} f^{0} .
\end{gathered}
$$

By the definition of $g_{k}$, with $k=n$, we have

$$
g_{n} \circ f=r_{n} g_{n} .
$$

Thus, for every non-negative integer $m$,

$$
g_{n} \circ f^{m+1}=r_{n}^{m+1} g_{n},
$$

that is,

$$
f^{n+m}-\left(\sum_{i \neq n} r_{i}\right) f^{n+m-1}+\ldots+(-1)^{n-1} r_{1} r_{2} \ldots r_{n-1} f^{m+1}=r_{n}^{m+1} g_{n},
$$

is a linear equation for $f^{n+m}, f^{n+m-1}, \ldots, f^{m+1}$. Similarly, for each fixed $k, \quad k=1,2, \ldots, n-1$, we get another linear equation. Thus we obtain a system of $n$ linear equations, expressed by

$$
A F=G,
$$

where $A$ is a matrix defined by (6), $F$ and $G$ are transposes of the vectors $\left(f^{n+m}, f^{n+m-1}, \ldots, f^{m+1}\right)$ and $\left(r_{1}^{m+1} g_{1}, r_{2}^{m+1} g_{2}, \ldots, r_{n}^{m+1} g_{n}\right)$, respectively. Applying repeatedly elementary linear transformations on the rows of $A$ we obtain

$$
\Delta=\operatorname{det} A=\prod_{i<j,=1}^{n}\left(r_{j}-r_{i}\right) \neq 0,
$$

i.e., $A$ is invertible. Now formula (5) is a direct consequence of Cramer's rule.

Corollary 1. Suppose that the polynomial $P_{n}$ in (2) has n pairwise different roots $r_{1}, \ldots, r_{n}$ and that $f: \mathbf{X} \rightarrow \mathbf{X}$ is a solution of a $k$-order equation of the form (1) whose characteristic polynomial $Q_{k}$ exactly divides $P_{n}$. Then $f^{n+m}$ is a sum of the suitable $k$ terms which appear in (5).

Proof. Since $Q_{k} \mid P_{n}$, we may assume without any loss of generality that the first $k$ numbers $r_{1}, r_{2}, \ldots, r_{k}$ are the $k$ roots of $Q_{k}$. Thus the function $f$ satisfies the equation $F_{k}\left(r_{1}, r_{2}, \ldots, r_{k}\right) f=0$. By Theorem 1 ,

$$
F_{n-1}\left(r_{1}, \ldots, r_{k}, \ldots, \check{r}_{i}, \ldots, r_{n}\right) f=0, \quad i=k+1, \ldots, n,
$$


that is, according to the notations in Theorem $2, g_{i}=0, \quad i=k+1, \ldots, n$. By Theorem 2,

$$
f^{n+m}=\frac{A_{11}}{\Delta} r_{1}^{m+1} g_{1}+\frac{A_{21}}{\Delta} r_{2}^{m+1} g_{2}+\ldots+\frac{A_{k 1}}{\Delta} r_{k}^{m+1} g_{k}, \quad m \geq 0,
$$

which completes the proof.

Remark 3. It is easy to verify that a solution $f: \mathbf{X} \rightarrow \mathbf{X}$ of ( 1 ) is oneto-one if $a_{0} \neq 0$; if moreover $\mathbf{X}=\mathbf{R}$ and $f$ is continuous then it is strictly monotone and onto. If $a_{0} \neq 0$ then, by (2), the characteristic polynomial of equation (1) has no zero root.

Obviously, if $a_{0} \neq 0$ and $f$ is onto then equations (1) and (3) are equivalent, respectively, to

$$
f^{-n}(x)=-\frac{a_{1}}{a_{0}} f^{-(n-1)}(x)+\ldots-\frac{a_{n-1}}{a_{0}} f^{-1}(x)+\frac{1}{a_{0}} x, \quad x \in \mathbf{X},
$$

and

$$
f^{-n}-\left(\sum_{i=1}^{n} s_{i}\right) f^{-(n-1)}+\left(\sum_{i<j}^{n} s_{i} s_{j}\right) f^{-(n-2)}+\ldots+(-1)^{n} s_{1} s_{2} \ldots s_{n} f^{0}=0,
$$

where $f^{-k}$ denotes the $k$-th iterate of $f^{-1}$ and $s_{i}=r_{i}^{-1}, i=1,2, \ldots, n$. In fact, in this case $f$ is invertible, maps $\mathbf{X}$ onto itself and satisfies (1). Usually (7) and (8) are called the dual equations of (1) and (3), respectively. The following result is the dual counterpart of Theorem 2 .

Theorem 3. Suppose that the hypotheses of Theorem 2 hold. If $f$ is onto and $a_{0} \neq 0$ in (1) then, for any integer $m \geq 0$,

$$
f^{-(n+m)}=\frac{\tilde{A}_{11}}{\tilde{\Delta}} s_{1}^{m+1} \tilde{g}_{1}+\frac{\tilde{A}_{21}}{\tilde{\Delta}} s_{2}^{m+1} \tilde{g}_{2}+\ldots+\frac{\tilde{A}_{n 1}}{\tilde{\Delta}} s_{n}^{m+1} \tilde{g}_{n},
$$

where $\tilde{g}_{i}, \tilde{\Delta}$ and $\tilde{A}_{i 1}, \quad i=1,2, . ., n$, are just modified $g_{i}, \Delta$ and $A_{i 1}, \quad i=$ $1,2, . ., n$, defined in Theorem 2 where $r_{j}$ is replaced by $s_{j}, \quad j=1,2, \ldots, n$, and $f$ is replaced by $f^{-1}$.

\section{Some properties of solutions}

Assume that $\mathbf{X}$ is a normed space, $a_{0} \neq 0$ and that the characteristic polynomial of equation (1) has $n$ pairwise different roots $r_{1}, r_{2}, \ldots, r_{n}$.

Corollary 2. Let $f: \mathbf{X} \rightarrow \mathbf{X}$ be a continuous solution of equation (1).

$1^{0}$ If $\left|r_{k}\right|<1$ for all $k=1, \ldots, n$, then $f^{k}$ approaches 0 as $k \rightarrow+\infty$; 
$2^{0}$ If $f$ is onto and $\left|r_{k}\right|>1$ for all $k=1, \ldots, n$, then $f^{k}$ approaches 0 as $k \rightarrow-\infty$;

$3^{0}$ In both cases 0 is a unique fixed point of $f$.

Proof. Letting $m \rightarrow+\infty$ in (5) gives $1^{0}$. Similarly $2^{0}$ is a consequence of the formula in Theorem 3 . To prove $3^{0}$ assume that $f\left(x_{0}\right)=x_{0}$ for some $x_{0} \neq 0$. From $(3)$,

$$
x_{0}-\left(\sum_{i=1}^{n} r_{i}\right) x_{0}+\left(\sum_{i<j}^{n} r_{i} r_{j}\right) x_{0}+\ldots+(-1)^{n} r_{1} r_{2} \ldots r_{n} x_{0}=0,
$$

that is, $\prod_{i=1}^{n}\left(1-r_{i}\right)=0$. Thus at least one of $r_{i}, \quad i=1,2, \ldots, n$, would be equal 1. This contradicts the hypotheses in $1^{0}$ and $2^{0}$. Therefore $f$ has no non-zero fixed point.

Now the relation $f(0)=0$ is an obvious consequence of the continuity of the function $f$.

In the next result we assume that $\mathbf{X}=\mathbf{R}$.

Corollary 3. Suppose that $f: \mathbf{R} \rightarrow \mathbf{R}$ is a strictly increasing and continuous solution of equation (1). The following results are true.

$1^{0}$ If $-1<r_{1}<\ldots<r_{n-1}<1<r_{n}$ or $r_{1}<-1<r_{2}<\ldots<r_{n}<1$, and if $f(x)<x$ for all $x>0$ and $f(x)>x$ for all $x<0$, then $f$ satisfies

$$
F_{n-1}\left(r_{1}, \ldots, r_{n-1}\right) f=0 \quad \text { or } \quad F_{n-1}\left(r_{2}, \ldots, r_{n}\right) f=0 .
$$

$2^{0}$ If $<r_{1}<\ldots<r_{n-1}<-1<r_{n}$ or $r_{1}<1<r_{2}<\ldots<r_{n}$, and if $f(x)>x$ for all $x>0$ and $f(x)<x$ for all $x<0$, then $f$ satisfies

$$
F_{n-1}\left(r_{1}^{-1}, \ldots, r_{n-1}^{-1}\right) f^{-1}=0 \quad \text { or } \quad F_{n-1}\left(r_{2}^{-1}, \ldots, r_{n}^{-1}\right) f^{-1}=0 .
$$

Proof. By similar arguments as in the last corollary it is easy to show that, in both cases, 0 is a unique fixed point of $f$ in $\mathbf{R}$. To prove $1^{0}$ assume that $-1<r_{1}<\ldots<r_{n-1}<1<r_{n}$ and take arbitrary $x>0$. Since $f$ is increasing, we have

$$
x>f(x)>f^{2}(x)>\ldots>f^{k}(x) \rightarrow 0, \quad \text { as } k \rightarrow+\infty, \quad x>0 .
$$

Similarly, for arbitrary $x<0$, we have

$$
x<f(x)<f^{2}(x)<\ldots<f^{k}(x) \rightarrow 0, \quad \text { as } k \rightarrow+\infty, \quad x>0 .
$$

By Theorem $2, g_{n}$ vanishes, i.e., $F_{n-1}\left(r_{1}, \ldots, r_{n-1}\right) f=0$ because $\left|r_{i}\right|^{k} \rightarrow$ $0, i=1,2, \ldots, n-1$, and $\left|r_{n}\right|^{k}$ does not as $k \rightarrow+\infty$. Similarly, applying Theorem 3 , we can prove $2^{0}$. 
Acknowledgement. The authors are grateful to the referees for their helpful comments.

\section{References}

[1] Fort, Jr., M.K., Continuous solutions of a functional equation, Ann. Polon. Math. 13 (1963), 205-211.

[2] Jarczyk, W., On an equation of linear iteration, Aequationes Math. 51 (1996), 303310.

[3] Jarczyk, W., Series of iterates summing up to the identity function, Grazer Math. Ber. 334 (1997), 153-162.

[4] Kuczma, M., Functional Equations in a Single Variable, Monografie Matematyczne 46, Warszawa, 1968.

[5] Kuczma, M., Choczewski, B. and Ger, R., Iterative Functional Equations, Encyclopedia Math. Appl. 32, Cambridge Univ. Press, 1990.

[6] Matkowski, J., The 26th International Symposium on Functional Equations, Remark 35, Aequationes Math. 37 (1989), 119-120.

[7] Mukherjea, A. and Ratti, J.S., On a functional equation involving iterates of a bijection on the unit interval, Nonlinear Anal. 7 (1983), 899-908.

[8] Nabeya, S., On the functional equation $f(p+q x+r f(x))=a+b x+c f(x)$, Aequationes Math. 11 (1974), 199-211.

[9] Tabor, J. and Tabor, J.,On a linear iterative equation, Results Math. 27 (1995), $412-421$.

[10] Zhang, W., Discussion on the differentiable solutions of the iterated equation $\sum_{i=1}^{n} \lambda_{i} f^{i}(x)=F(x)$, Nonlinear Anal. 15 (1990), 387-398.

[11] Zhang, W. and Baker, J.A., Continuous solutions of a polynomial-like iterative equation with variable coefficients, Waterloo Rep. Fac. 11 (1994), University of Waterloo, Ontario, Canada.

JANUSZ MATKOWSKI

Institute OF MATHEMATICS

Pedagogical University

PL. SŁOWIAŃSKI 9

65-069 Zielona Góra, Poland

AND

Institute of Mathematics

Silesian UnIVERSITY

Katowice, Poland
WEINIAN ZHANG

Department of MATHEMATiCs

SiCHUAN UNION UNIVERSITY

East Area, Chengdu 610064

P.R. CHINA 